\begin{tabular}{|c|c|c|}
\hline \multirow{3}{*}{$\begin{array}{r}\text { Case Reports in } \\
\text { Gastroenterology }\end{array}$} & \multirow{2}{*}{\multicolumn{2}{|c|}{ Case Rep Gastroenterol 2015;9:317-326 }} \\
\hline & & \\
\hline & $\begin{array}{l}\text { DOI: 10.1159/000441387 } \\
\text { Publisnea ontune: Uctober 17, } 2015\end{array}$ & $\begin{array}{l}\text { (c) } 2015 \text { The Author(s) } \\
\text { Published by S. Karger AG, Basel } \\
\text { 1662-0631/15/0093-0317 } \$ 39.50 / 0 \\
\text { www.karger.com/crg }\end{array}$ \\
\hline & $\begin{array}{l}\text { This article is licensed under the } C \\
\text { International License (CC BY-NC) (h } \\
\text { Usage and distribution for commerci }\end{array}$ & $\begin{array}{l}\text { mons Attribution-NonCommercial } \\
\text { rger.com/Services/OpenAccessLicens } \\
\text { equires written permission. }\end{array}$ \\
\hline
\end{tabular}

\title{
Recent Trend of Hepatitis E Virus Infection in Chiba Area, Japan: 3 of 5 Cases with Rheumatoid Arthritis
}

\author{
Tatsuo Kanda ${ }^{a}$ Shin Yasui ${ }^{a}$ Masato Nakamura ${ }^{a}$ Makoto Arai $^{a}$ \\ Reina Sasaki $^{a}$ Yuki Haga $^{a}$ Shuang $\mathrm{Wu}^{\mathrm{a}}$ Shingo Nakamoto ${ }^{\mathrm{b}}$ \\ Hiroaki Okamoto ${ }^{c}$ Osamu Yokosuka ${ }^{a}$ \\ Departments of ${ }^{\mathrm{a}}$ Gastroenterology and Nephrology, and ${ }^{\mathrm{b}}$ Molecular Virology, Graduate \\ School of Medicine, Chiba University, Chiba, and ' ${ }^{\mathrm{C} D i v i s i o n}$ of Virology, Department of \\ Infection and Immunity, Jichi Medical University School of Medicine, Tochigi, Japan
}

\section{Key Words}

Autoimmune hepatitis · Hepatitis E virus - Rheumatoid arthritis · IgA anti-hepatitis E virus antibody

\begin{abstract}
Hepatitis $E$ virus (HEV) infection is an emerging health concern in developing and developed countries, such as Japan. Five cases have recently been diagnosed as hepatitis E. Of interest, 3 of them had rheumatoid arthritis (RA), although a previous study demonstrated a lack of association between HEV and RA. One of the other patients developed autoimmune hepatitis and was successfully treated with corticosteroids approximately 150 days after the diagnosis of hepatitis E. In RA patients with liver dysfunction, the presence of HEV infection should be evaluated immediately because these patients are often relatively old. Further investigation of the association between HEV and autoimmune hepatitis is needed.
\end{abstract}

(C) 2015 The Author(s)

Published by S. Karger AG, Basel

\section{Introduction}

Hepatitis E virus (HEV) is a non-enveloped, single-stranded positive-sense RNA virus of approximately $7.2 \mathrm{~kb}$ [1]. HEV infection is transmitted primarily through the fecal-oral route $[1,2]$. HEV infection may lead to acute hepatitis, including acute liver failure [3], and chronic 
Kanda et al.: Recent Trend of Hepatitis E Virus Infection in Chiba Area, Japan: 3 of 5 Cases with Rheumatoid Arthritis

hepatitis in organ transplant recipients [4]. HEV infection is recognized as a serious health problem in developing and in developed countries [1-6].

In Japan, 3.4\% of qualified blood donors were positive for immunoglobulin (Ig)G antiHEV antibodies [7]. Our previous study showed that $23 \%$ of the indigenous Japanese population, including individuals over 50 years of age [8], were positive for IgG anti-HEV antibodies. This suggests that Japanese subjects are susceptible to HEV infection and demonstrates the importance of controlling and investigating HEV infection due to the lack of availability of an HEV vaccine in Japan. In 2011, IgA anti-HEV antibody tests were available for the diagnosis of HEV by the Japanese national health insurance system [9].

In patients with rheumatoid arthritis (RA), acute liver injury associated with autoimmune hepatitis and drug-induced liver injury (DILI), reactivation of hepatitis B virus (HBV), and liver dysfunction caused by other reasons are occasionally observed [10,11]. Here, we report 5 patients who were recently identified to have HEV infection, 3 of whom were also diagnosed with RA.

\section{Case Report}

Five cases of HEV infection were observed in our hospital between January 2014 and April 2015. HEV infection was diagnosed by positivity for IgA anti-HEV antibody [9]. The clinical features of the 5 patients in the present study are briefly described in table 1 . Case 5 visited our hospital approximately 150 days after onset. All patients were over 50 years old, and 4 of them were female patients. Three cases visited a hospital for their RA, and they took several types of medicine to treat the RA. Cases 2 and 4 drank alcohol ( 40 and $20 \mathrm{~g}$ daily, respectively). Autoantibodies were positive in 3 cases (cases 1, 2, and 5). The clinical courses and laboratory data from the first visit are shown in figure 1 and table 2, respectively.

Case 1

A 64-year-old female who was diagnosed with RA 9 years ago and who received treatment in another hospital was referred to our hospital with general fatigue and liver dysfunction (table 1; fig. 1a). Laboratory data on the first visit to our hospital showed an improved liver function test (table 2a). Her height and body weight were $147 \mathrm{~cm}$ and $44 \mathrm{~kg}$, respectively. She was positive for HEV genotype 3 RNA and IgA anti-HEV antibody (fig. 1a). She was also positive for anti-mitochondrial antibody, and a liver biopsy showed Scheuer stage I of primary biliary cirrhosis (PBC) (fig. 2a, b). We ultimately diagnosed her as having HEV infection and PBC, although we initially doubted DILI.

Case 2

A 59-year-old male with a diagnosis of alcoholic liver disease was referred to our hospital with general fatigue and marked liver dysfunction (tables $1,2 \mathrm{~b}$; fig. 1b). His height and body weight were $176 \mathrm{~cm}$ and $69 \mathrm{~kg}$, respectively. He was positive for HEV genotype 3 RNA and IgA anti-HEV antibody (fig. 1b). We diagnosed him as having HEV infection. After admission to our hospital, he was given bed rest and peripheral parenteral nutrition, and his condition improved. This patient had consumed deer meat approximately 1 month before admission.

Case 3

A 74-year-old female who was diagnosed as having RA 20 years ago and was treated in a different hospital was referred to our hospital with general fatigue and liver dysfunction 
Kanda et al.: Recent Trend of Hepatitis E Virus Infection in Chiba Area, Japan: 3 of 5 Cases with Rheumatoid Arthritis

(tables 1, 2c; fig. 1c). Her height and body weight were $154 \mathrm{~cm}$ and $54 \mathrm{~kg}$, respectively. Because she was treated with tofacitinib only 2 months previously, we initially doubted that DILI was caused by this drug. However, she was positive for HEV genotype 3 RNA and IgA anti-HEV antibody (fig. 1c). We diagnosed her as having HEV infection.

Case 4

A 52-year-old female who was diagnosed with RA 4 years ago and whose RA was treated in another hospital was referred to our hospital with general fatigue and liver dysfunction (tables 1, 2d; fig. 1d). Her height and body weight were $152 \mathrm{~cm}$ and $50 \mathrm{~kg}$, respectively. She was positive for HEV genotype 3 RNA and IgA anti-HEV antibody (fig. 1d). We diagnosed her as having HEV infection. After admission to our hospital, she was given bed rest and peripheral parenteral nutrition, and her condition improved. A liver biopsy confirmed acute hepatitis (fig. 2c, d).

Case 5

A 77-year-old female who was diagnosed with HEV infection based on positivity for IgA anti-HEV antibody approximately 150 days before admission to our hospital was referred to our hospital with general fatigue and liver dysfunction (tables 1, 2e; fig. 1e). Her height and body weight were $144 \mathrm{~cm}$ and $50 \mathrm{~kg}$, respectively. She was positive for antinuclear antibody, and her IgG was elevated. A liver biopsy showed typical characteristics of autoimmune hepatitis (fig. 2e-g). We began corticosteroid therapy, and her liver tests improved; however, her positivity for IgA anti-HEV persisted 9 months after onset. Upon admission to our hospital, although HEV RNA was negative, we diagnosed her as having HEV infection according to the changes of titers of anti-HEV antibodies (fig. 1e). An updated (1999) scoring system for the diagnosis of autoimmune hepatitis [12] indicated probable autoimmune hepatitis (score: 16) because viral hepatitis by HEV was not completely ruled out on this score system.

\section{Discussion}

In the current study, we presented 5 cases with HEV infection. The mean age of these patients was $65 \pm 11$ years, 4 patients were female, and 3 had RA. Two patients were over 65 years of age, and 1 of these 2 patients was over 65 years old and had RA. Only 1 male patient without RA reported consumption of deer meet before onset. In the other 4 patients, the infectious sources of HEV were unknown.

Pischke et al. [13] reported that patients with autoimmune hepatitis, but not RA or $\mathrm{HBV} / \mathrm{HCV}$ patients, are more likely to test positive for anti-HEV. They reported that only 4 of $114(3.5 \%)$ RA patients were positive for HEV-specific antibodies, similar to healthy individuals [11 of $537(2.0 \%)]$. However, we found that 3 of 5 patients with HEV infection had RA. The present study suggests that it is important to rule out HEV infection in RA patients with liver dysfunction.

Although an initial diagnosis of HEV infection was made based on the positivity for IgA anti-HEV antibody in case 5, she was also diagnosed as having autoimmune hepatitis based on a subsequent liver biopsy (fig. 2e). Although a corticosteroid was administered and her liver dysfunction improved, positivity for IgA anti-HEV antibody persisted for 9 months after onset. It is well known that autoimmune hepatitis rarely follows acute viral hepatitis [14]. Inagaki et al. [15] reported that a 65-year-old female with HEV RNA was diagnosed as having probable autoimmune hepatitis and was successfully treated with prednisolone. Nagasaki et al. [16] also recommended that HEV infection should be ruled out in the cases with acute 
Kanda et al.: Recent Trend of Hepatitis E Virus Infection in Chiba Area, Japan: 3 of 5 Cases with Rheumatoid Arthritis

cryptogenic hepatitis, including autoimmune hepatitis. Three of the cases described in the present study were positive for autoantibodies. Careful attention should be given to these features [17]. Together, HEV infection could trigger autoimmune hepatitis in some cases.

Recently, reports about HBV reactivation in RA patients treated with newer biological drugs like tocilizumab and abatacept have been increasing [18]. A remarkably high incidence of tuberculosis in RA patients treated with TNF- $\alpha$ antagonists has been reported [19]. In the present study, 3 patients with RA were well controlled and had stable activity of RA. Of interest, case 3 took tofacitinib. Further studies will be needed.

RA patients with liver dysfunction are treated with several medications, and DILI should also be ruled out (table 1), as well as viral hepatitis. In conclusion, due, in part, to the availability of the IgA anti-HEV antibody, we have recently diagnosed 5 cases as being positive for HEV. In RA patients with liver dysfunction, HEV infection should also be ruled out using the IgA anti-HEV antibody, because these patients include older patients. Further studies regarding the association between HEV and autoimmune hepatitis are needed. The present study also showed that HEV infection is an important emerging health concern.

\section{Acknowledgements}

This research was (partially) supported by the Research Program on Hepatitis from Japan Agency for Medical Research and Development (AMED).

\section{Statement of Ethics}

The authors have no ethical conflicts to disclose.

\section{Disclosure Statement}

Prof. Osamu Yokosuka reports receiving grant support from Chugai Pharmaceutical, Bayer, MSD, Daiichi-Sankyo, Tanabe-Mitsubishi, Bristol-Myers Squibb, Taiho Pharmaceutical, and Gilead Sciences. The other authors have no conflict of interest statement.

\section{References}

1 Takahashi M, Okamoto H: Features of hepatitis E virus infection in humans and animals in Japan. Hepatol Res 2014;44:43-58.

2 Ray R, Aggarwal R, Salunke PN, Mehrotra NN, Talwar GP, Naik SR: Hepatitis E virus genome in stools of hepatitis patients during large epidemic in north India. Lancet 1991;338:783-784.

-3 Acharya SK, Dasarathy S, Kumer TL, Sushma S, Prasanna KS, Tandon A, Sreenivas V, Nijhawan S, Panda SK, Nanda SK, Irshad M, Joshi YK, Duttagupta S, Tandon RK, Tandon BN: Fulminant hepatitis in a tropical population: clinical course, cause, and early predictors of outcome. Hepatology 1996;23:1448-1455.

-4 Kamar N, Selves J, Mansuy JM, Ouezzani L, Péron JM, Guitard J, Cointault O, Esposito L, Abravanel F, Danjoux M, Durand D, Vinel JP, Izopet J, Rostaing L: Hepatitis E virus and chronic hepatitis in organ-transplant recipients. N Engl J Med 2008;358:811-817.

5 Takahashi K, Iwata K, Watanabe N, Hatahara T, Ohta Y, Baba K, Mishiro S: Full-genome nucleotide sequence of a hepatitis E virus strain that may be indigenous to Japan. Virology 2001;287:9-12.

-6 Okamoto H, Takahashi M, Nishizawa T, Fukai K, Muramatsu U, Yoshikawa A: Analysis of the complete genome of indigenous swine hepatitis E virus isolated in Japan. Biochem Biophys Res Commun 2001;289:929-936. 
Kanda et al.: Recent Trend of Hepatitis E Virus Infection in Chiba Area, Japan: 3 of 5 Cases with Rheumatoid Arthritis

7 Takeda H, Matsubayashi K, Sakata H, Sato S, Kato T, Hino S, Tadokoro K, Ikeda H: A nationwide survey for prevalence of hepatitis E virus antibody in qualified blood donors in Japan. Vox Sang 2010;99:307-313.

-8 Yan J, Kanda T, Wu S, Imazeki F, Yokosuka O: Hepatitis A, B, C and E virus markers in Chinese residing in Tokyo, Japan. Hepatol Res 2012;42:974-981.

-9 Takahashi M, Kusakai S, Mizuo H, Suzuki K, Fujimura K, Masuko K, Sugai Y, Aikawa T, Nishizawa T, Okamoto $\mathrm{H}$ : Simultaneous detection of immunoglobulin A (IgA) and IgM antibodies against hepatitis E virus (HEV) is highly specific for diagnosis of acute HEV infection. J Clin Microbiol 2005;43:49-56.

10 Takahashi A, Abe K, Yokokawa J, Iwadate H, Kobayashi H, Watanabe H, Irisawa A, Ohira H: Clinical features of liver dysfunction in collagen diseases. Hepatol Res 2010;40:1092-1097.

11 Nakamoto S, Kanda T, Nakaseko C, Sakaida E, Ohwada C, Takeuchi M, Takeda Y, Mimura N, Iseki T, Wu S, Arai M, Imazeki F, Saito K, Shirasawa H, Yokosuka O: Reactivation of hepatitis B virus in hematopoietic stem cell transplant recipients in Japan: efficacy of nucleos(t)ide analogues for prevention and treatment. Int J Mol Sci 2014;15:21455-21467.

-12 Alvarez F, Berg PA, Bianchi FB, Bianchi L, Burroughs AK, Cancado EL, Chapman RW, Cooksley WG, Czaja AJ, Desmet VJ, Donaldson PT, Eddleston AL, Fainboim L, Heathcote J, Homberg JC, Hoofnagle JH, Kakumu S, Krawitt EL, Mackay IR, MacSween RN, Maddrey WC, Manns MP, McFarlane IG, Meyer zum Büschenfelde KH, Zeniya M, et al: International Autoimmune Hepatitis Group Report: review of criteria for diagnosis of autoimmune hepatitis. J Hepatol 1999;31:929-938.

13 Pischke S, Gisa A, Suneetha PV, Wiegand SB, Taubert R, Schlue J, Wursthorn K, Bantel H, Raupach R, Bremer B, Zacher BJ, Schmidt RE, Manns MP, Rifai K, Witte T, Wedemeyer H: Increased HEV seroprevalence in patients with autoimmune hepatitis. PLoS One 2014;9:e85330.

14 Mikata R, Yokosuka O, Imazeki F, Fukai K, Kanda T, Saisho H: Prolonged acute hepatitis A mimicking autoimmune hepatitis. World J Gastroenterol 2005;11:3791-3793.

15 Inagaki Y, Oshiro Y, Hasegawa N, Fukuda K, Abei M, Nishi M, Okamoto H, Ohkohchi N: Clinical features of hepatitis E virus infection in Ibaraki, Japan: autochthonous hepatitis $\mathrm{E}$ and acute-on-chronic liver failure. Tohoku J Exp Med 2015;235:275-282.

16 Nagasaki F, Ueno Y, Mano Y, Igarashi T, Yahagi K, Niitsuma H, Okamoto H, Shimosegawa T: A patient with clinical features of acute hepatitis E viral infection and autoimmune hepatitis. Tohoku J Exp Med 2005;206:173-179.

17 Nagasaki F, Ueno Y, Kanno N, Okamoto H, Shimosegawa T: A case of acute hepatitis with positive autoantibodies who actually had hepatitis E virus infection. Hepatol Res 2005;32:134-137.

-18 Nard FD, Todoerti M, Grosso V, Monti S, Breda S, Rossi S, Montecucco C, Caporali R: Risk of hepatitis B virus reactivation in rheumatoid arthritis patients undergoing biologic treatment: extending perspective from old to newer drugs. World J Hepatol 2015;7:344-361.

19 Yasui K: Immunity against Mycobacterium tuberculosis and the risk of biologic anti-TNF- $\alpha$ reagents. Pediatr Rheumatol Online J 2014;12:45.

20 Inoue J, Takahashi M, Yazaki Y, Tsuda F, Okamoto H: Development and validation of an improved RT-PCR assay with nested universal primers for detection of hepatitis $E$ virus strains with significant sequence divergence. J Virol Methods 2006;137:325-333. 
Kanda et al.: Recent Trend of Hepatitis E Virus Infection in Chiba Area, Japan: 3 of 5 Cases with Rheumatoid Arthritis

Table 1. Clinical features of 5 patients with HEV infection

\begin{tabular}{|c|c|c|c|c|c|}
\hline & Case 1 & Case 2 & Case 3 & Case 4 & Case 5 \\
\hline Age, years/gender & $64 /$ female & $59 /$ male & 74/female & $52 /$ female & $77 /$ female \\
\hline$\overline{\text { Duration, days }^{1}}$ & 19 & 1 & 7 & 9 & $\sim 150$ \\
\hline Underlying diseases & RA & ALD & RA & RA & hypertension \\
\hline Symptom(s) & fatigue & fever, fatigue & fatigue & epigastric discomfort & fatigue \\
\hline Max. AST, IU/l & 202 & 1,953 & 842 & 813 & 793 \\
\hline Max. ALT, IU/l & 527 & 1,944 & 753 & 973 & 850 \\
\hline $\begin{array}{l}\text { Max. total bilirubin, } \\
\mathrm{mg} / \mathrm{dl}\end{array}$ & 0.6 & 5.4 & 0.7 & 1.1 & 1.7 \\
\hline Min. PT, \% & 126 & 100 & 114 & 84 & 56 \\
\hline Source of infection & unknown & deer meat & unknown & unknown & unknown \\
\hline ANA, -fold & 80 & 160 & 80 & 40 & 320 \\
\hline ASMA, -fold/AMA M2 & $80 / 14.6$ & neg./neg. & neg./neg. & neg./neg. & neg./neg. \\
\hline Medicine before onset & $\begin{array}{l}\text { bucillamine, } \\
\text { loxoprofen, } \\
\text { methotrexate, folic } \\
\text { acid, alendronate, } \\
\text { eldecalcitol, } \\
\text { rabeprazole sodium, } \\
\text { magnesium oxide, } \\
\text { UDCA }\end{array}$ & $\begin{array}{l}\text { acetylsalicylic } \\
\text { acid }\end{array}$ & $\begin{array}{l}\text { tofacitinib, prednisolone, } \\
\text { loxoprofen, salazosulfa- } \\
\text { pyridine, eldecalcitol, } \\
\text { tocopherol nicotinate, } \\
\text { methylcobalamin, } \\
\text { teprenone, limaprost } \\
\text { alfadex }\end{array}$ & $\begin{array}{l}\text { methotrexate, } \\
\text { triamcinolone, folic } \\
\text { acid, pregabalin, } \\
\text { roxatidine acetate } \\
\text { hydrochloride, } \\
\text { neurotropin, } \\
\text { amoxapine, sulpiride, } \\
\text { etizolam }\end{array}$ & $\begin{array}{l}\text { nifedipine, can- } \\
\text { desartan cilexetil, } \\
\text { doxazosin, } \\
\text { famotidine, } \\
\text { rebamipide, } \\
\text { UDCA }\end{array}$ \\
\hline
\end{tabular}

$\mathrm{ALD}=$ Alcoholic liver disease; $\mathrm{AST}=$ aspartate transaminase ALT = alanine transaminase; Max. $=$ maximum; Min. $=$ minimum; $\mathrm{PT}=$ prothrombin time; $\mathrm{ANA}=$ anti-nuclear antibody; $\mathrm{ASMA}=$ anti-smooth muscle antibody; $\mathrm{AMA}=$ anti-mitochondrial antibody; neg. = negative; UDCA = ursodeoxycholic acid. ${ }^{1}$ Duration between onset and visit to the hospital. 
Kanda et al.: Recent Trend of Hepatitis E Virus Infection in Chiba Area, Japan: 3 of 5 Cases with Rheumatoid Arthritis

Table 2. Laboratory data for 5 patients with HEV infection on their first visit

\begin{tabular}{|c|c|c|c|c|c|}
\hline Category & Units & Category & Units & Category & Units \\
\hline \multicolumn{6}{|l|}{ a Case 1} \\
\hline AST & $22 \mathrm{IU} / \mathrm{l}$ & WBC & $6,600 / \mathrm{mm}^{3}$ & IgM HA & $(-)$ \\
\hline ALT & $46 \mathrm{IU} / \mathrm{l}$ & $\mathrm{RBC}$ & $419 \times 10^{4} / \mathrm{mm}^{3}$ & IgM HBc & $(-)$ \\
\hline G-GTP & $35 \mathrm{IU} / \mathrm{l}$ & $\mathrm{Hb}$ & $13.2 \mathrm{~g} / \mathrm{dl}$ & HBsAg & $(-)$ \\
\hline ALP & $185 \mathrm{IU} / \mathrm{l}$ & Hct & $38.5 \%$ & HBsAb & $(-)$ \\
\hline LDH & 172 IU/l & Platelets & $301 \times 10^{3} / \mathrm{mm}^{3}$ & $\mathrm{HBcAb}$ & $(-)$ \\
\hline $\mathrm{TP}$ & $7.5 \mathrm{~g} / \mathrm{dl}$ & Eosinophils & $1.5 \%$ & HBV DNA & $(-)$ \\
\hline ALB & $3.9 \mathrm{~g} / \mathrm{dl}$ & РТ\% & $126 \%$ & $\mathrm{HCV} \mathrm{Ab}$ & $(-)$ \\
\hline T. Bil & $0.5 \mathrm{mg} / \mathrm{dl}$ & PT-INR & 0.91 & HCV RNA & $(-)$ \\
\hline D. Bil & $0.1 \mathrm{mg} / \mathrm{dl}$ & ESR & $25 \mathrm{~mm} / \mathrm{h}$ & IgA HEV & $(+)$ \\
\hline T. CHO & $187 \mathrm{mg} / \mathrm{dl}$ & $\mathrm{HbA1C}$ & $5.0 \%$ & ANA & $\times 80$ \\
\hline UA & $5.0 \mathrm{mg} / \mathrm{dl}$ & IgG & $1,580 \mathrm{mg} / \mathrm{dl}$ & ASMA & $\times 80$ \\
\hline UN & $13 \mathrm{mg} / \mathrm{dl}$ & IgM & 337 mg/dl & AMA M2 & $14.6(+)$ \\
\hline Cre & $0.46 \mathrm{mg} / \mathrm{dl}$ & $\operatorname{IgA}$ & $299 \mathrm{mg} / \mathrm{dl}$ & aLKM1 & $(-)$ \\
\hline CRP & $0.1 \mathrm{mg} / \mathrm{dl}$ & TSH & $1.086 \mu \mathrm{IU} / \mathrm{ml}$ & AFP & $3.2 \mathrm{ng} / \mathrm{ml}$ \\
\hline \multicolumn{6}{|l|}{ b Case 2} \\
\hline AST & 1,953 IU/l & WBC & $3,900 / \mathrm{mm}^{3}$ & IgM HA & $(-)$ \\
\hline ALT & 1,944 IU/l & $\mathrm{RBC}$ & $473 \times 10^{4} / \mathrm{mm}^{3}$ & IgM HBc & $(-)$ \\
\hline G-GTP & $401 \mathrm{IU} / \mathrm{l}$ & $\mathrm{Hb}$ & $14.6 \mathrm{~g} / \mathrm{dl}$ & HBsAg & $(-)$ \\
\hline ALP & $710 \mathrm{IU} / \mathrm{l}$ & Hct & $43.4 \%$ & HBsAb & $(-)$ \\
\hline LDH & 1,293 IU/l & Platelets & $127 \times 10^{3} / \mathrm{mm}^{3}$ & $\mathrm{HBcAb}$ & $(-)$ \\
\hline $\mathrm{TP}$ & $7.4 \mathrm{~g} / \mathrm{dl}$ & РT\% & $111 \%$ & HBV DNA & $(-)$ \\
\hline ALB & $3.9 \mathrm{~g} / \mathrm{dl}$ & PT-INR & 1.01 & $\mathrm{HCV} A b$ & $(-)$ \\
\hline T. Bil & $4.5 \mathrm{mg} / \mathrm{dl}$ & ESR & $35 \mathrm{~mm} / \mathrm{h}$ & HCV RNA & $(-)$ \\
\hline D. Bil & $3.2 \mathrm{mg} / \mathrm{dl}$ & $\mathrm{HbA1C}$ & $5.4 \%$ & IgA HEV & $(+)$ \\
\hline T. CHO & $197 \mathrm{mg} / \mathrm{dl}$ & IgG & $1,928 \mathrm{mg} / \mathrm{dl}$ & ANA & $\times 160$ \\
\hline UA & $5.6 \mathrm{mg} / \mathrm{dl}$ & IgM & $259 \mathrm{mg} / \mathrm{dl}$ & ASMA & $(-)$ \\
\hline UN & $20 \mathrm{mg} / \mathrm{dl}$ & IgA & $259 \mathrm{mg} / \mathrm{dl}$ & AMA & $(-)$ \\
\hline Cre & $0.79 \mathrm{mg} / \mathrm{dl}$ & TSH & $3.604 \mu \mathrm{IU} / \mathrm{ml}$ & HIV & $(-)$ \\
\hline CRP & $2.2 \mathrm{mg} / \mathrm{dl}$ & HA & $71 \mathrm{ng} / \mathrm{ml}$ & AFP & $26.5 \mathrm{ng} / \mathrm{ml}$ \\
\hline \multicolumn{6}{|l|}{ c Case 3} \\
\hline AST & $262 \mathrm{IU} / \mathrm{l}$ & WBC & $12,800 / \mathrm{mm}^{3}$ & IgM HA & $(-)$ \\
\hline ALT & $444 \mathrm{IU} / \mathrm{l}$ & $\mathrm{RBC}$ & $386 \times 10^{4} / \mathrm{mm}^{3}$ & IgM HBc & $(-)$ \\
\hline G-GTP & $68 \mathrm{IU} / \mathrm{l}$ & $\mathrm{Hb}$ & $12.2 \mathrm{~g} / \mathrm{dl}$ & HBsAg & $(-)$ \\
\hline ALP & $229 \mathrm{IU} / \mathrm{l}$ & Hct & $36.9 \%$ & HBV DNA & $(-)$ \\
\hline LDH & $310 \mathrm{IU} / \mathrm{l}$ & Platelets & $286 \times 10^{3} / \mathrm{mm}^{3}$ & $\mathrm{HCV} A b$ & $(-)$ \\
\hline $\mathrm{TP}$ & $7.4 \mathrm{~g} / \mathrm{dl}$ & Eosinophils & $0.2 \%$ & HCV RNA & $(-)$ \\
\hline ALB & $4.1 \mathrm{~g} / \mathrm{dl}$ & РT\% & $114 \%$ & IgA HEV & $(+)$ \\
\hline T. Bil & $0.6 \mathrm{mg} / \mathrm{dl}$ & PT-INR & 1.02 & ANA & $\times 80$ \\
\hline D. Bil & $0.1 \mathrm{mg} / \mathrm{dl}$ & ESR & $27 \mathrm{~mm} / \mathrm{h}$ & ASMA & $(-)$ \\
\hline T. CHO & $200 \mathrm{mg} / \mathrm{dl}$ & $\mathrm{HbA1C}$ & $5.4 \%$ & AMA & $(-)$ \\
\hline UA & $6.4 \mathrm{mg} / \mathrm{dl}$ & IgG & $1,551 \mathrm{mg} / \mathrm{dl}$ & & \\
\hline UN & $19 \mathrm{mg} / \mathrm{dl}$ & IgM & $187 \mathrm{mg} / \mathrm{dl}$ & & \\
\hline Cre & $0.68 \mathrm{mg} / \mathrm{dl}$ & $\operatorname{IgA}$ & $145 \mathrm{mg} / \mathrm{dl}$ & & \\
\hline CRP & $3.4 \mathrm{mg} / \mathrm{dl}$ & TSH & $1.017 \mu \mathrm{IU} / \mathrm{ml}$ & & \\
\hline \multicolumn{6}{|l|}{ d Case 4} \\
\hline AST & $565 \mathrm{IU} / \mathrm{l}$ & WBC & $4,200 / \mathrm{mm}^{3}$ & IgM HA & $(-)$ \\
\hline ALT & 973 IU/l & $\mathrm{RBC}$ & $411 \times 10^{4} / \mathrm{mm}^{3}$ & IgM HBc & $(-)$ \\
\hline G-GTP & $506 \mathrm{IU} / \mathrm{l}$ & $\mathrm{Hb}$ & $14.1 \mathrm{~g} / \mathrm{dl}$ & HBsAg & $(-)$ \\
\hline ALP & 1,214 IU/l & Hct & $41.0 \%$ & HBV DNA & $(-)$ \\
\hline
\end{tabular}


Kanda et al.: Recent Trend of Hepatitis E Virus Infection in Chiba Area, Japan: 3 of 5 Cases with Rheumatoid Arthritis

\begin{tabular}{|c|c|c|c|c|c|}
\hline LDH & $374 \mathrm{IU} / \mathrm{l}$ & Platelets & $327 \times 10^{3} / \mathrm{mm}^{3}$ & $\mathrm{HCV} A b$ & $(-)$ \\
\hline ТP & $7.9 \mathrm{~g} / \mathrm{dl}$ & Eosinophils & $0.7 \%$ & HCV RNA & $(-)$ \\
\hline ALB & $4.2 \mathrm{~g} / \mathrm{dl}$ & РТ\% & $108 \%$ & IgA HEV & $(+)$ \\
\hline T. Bil & $4.3 \mathrm{mg} / \mathrm{dl}$ & PT-INR & 1.00 & ANA & $\times 40(-)$ \\
\hline D. Bil & $3.3 \mathrm{mg} / \mathrm{dl}$ & ESR & $22 \mathrm{~mm} / \mathrm{h}$ & ASMA & $(-)$ \\
\hline T. CHO & $161 \mathrm{mg} / \mathrm{dl}$ & $\mathrm{HbA1C}$ & $4.9 \%$ & AMA M2 & $1.6(-)$ \\
\hline UA & $3.7 \mathrm{mg} / \mathrm{dl}$ & $\operatorname{IgG}$ & $1,552 \mathrm{mg} / \mathrm{dl}$ & & \\
\hline UN & $8 \mathrm{mg} / \mathrm{dl}$ & IgM & $211 \mathrm{mg} / \mathrm{dl}$ & & \\
\hline Cre & $0.53 \mathrm{mg} / \mathrm{dl}$ & $\operatorname{IgA}$ & $178 \mathrm{mg} / \mathrm{dl}$ & & \\
\hline CRP & $1.1 \mathrm{mg} / \mathrm{dl}$ & TSH & $0.743 \mu \mathrm{IU} / \mathrm{ml}$ & & \\
\hline \multicolumn{6}{|c|}{ e Case 5} \\
\hline AST & $209 \mathrm{IU} / \mathrm{l}$ & WBC & $9,200 / \mathrm{mm}^{3}$ & IgM HA & $(-)$ \\
\hline ALT & $227 \mathrm{IU} / \mathrm{l}$ & $\mathrm{RBC}$ & $381 \times 10^{4} / \mathrm{mm}^{3}$ & IgM HBc & $(-)$ \\
\hline G-GTP & $56 \mathrm{IU} / \mathrm{l}$ & $\mathrm{Hb}$ & $12.5 \mathrm{~g} / \mathrm{dl}$ & HBsAg & $(-)$ \\
\hline ALP & $723 \mathrm{IU} / \mathrm{l}$ & Hct & $37.5 \%$ & HBV DNA & $(-)$ \\
\hline $\mathrm{LDH}$ & $338 \mathrm{IU} / \mathrm{l}$ & Platelets & $132 \times 10^{3} / \mathrm{mm}^{3}$ & $\mathrm{HCV}$ Ab & $(-)$ \\
\hline TP & $7.1 \mathrm{~g} / \mathrm{dl}$ & Eosinophils & $0.5 \%$ & HCV RNA & $(-)$ \\
\hline ALB & $2.5 \mathrm{~g} / \mathrm{dl}$ & РT\% & $60 \%$ & IgA HEV & $(+)$ \\
\hline T. Bil & $1.5 \mathrm{mg} / \mathrm{dl}$ & PT-INR & 1.27 & HEV RNA & $(-)$ \\
\hline D. Bil & $0.4 \mathrm{mg} / \mathrm{dl}$ & ESR & $20 \mathrm{~mm} / \mathrm{h}$ & IgG HEV & $(+)$ \\
\hline T. CHO & $146 \mathrm{mg} / \mathrm{dl}$ & HbA1C & $4.9 \%$ & ANA & $\times 320(+)$ \\
\hline UA & $5.1 \mathrm{mg} / \mathrm{dl}$ & IgG & $2,777 \mathrm{mg} / \mathrm{dl}$ & ASMA & $(-)$ \\
\hline UN & $10 \mathrm{mg} / \mathrm{dl}$ & IgM & $163 \mathrm{mg} / \mathrm{dl}$ & AMA & $(-)$ \\
\hline Cre & $0.54 \mathrm{mg} / \mathrm{dl}$ & IgA & $560 \mathrm{mg} / \mathrm{dl}$ & ACE & 31.9 \\
\hline CRP & $0.8 \mathrm{mg} / \mathrm{dl}$ & TSH & $1.151 \mu \mathrm{IU} / \mathrm{ml}$ & NH3 & $82 \mu \mathrm{g} / \mathrm{ml}$ \\
\hline
\end{tabular}


Kanda et al.: Recent Trend of Hepatitis E Virus Infection in Chiba Area, Japan: 3 of 5 Cases with Rheumatoid Arthritis
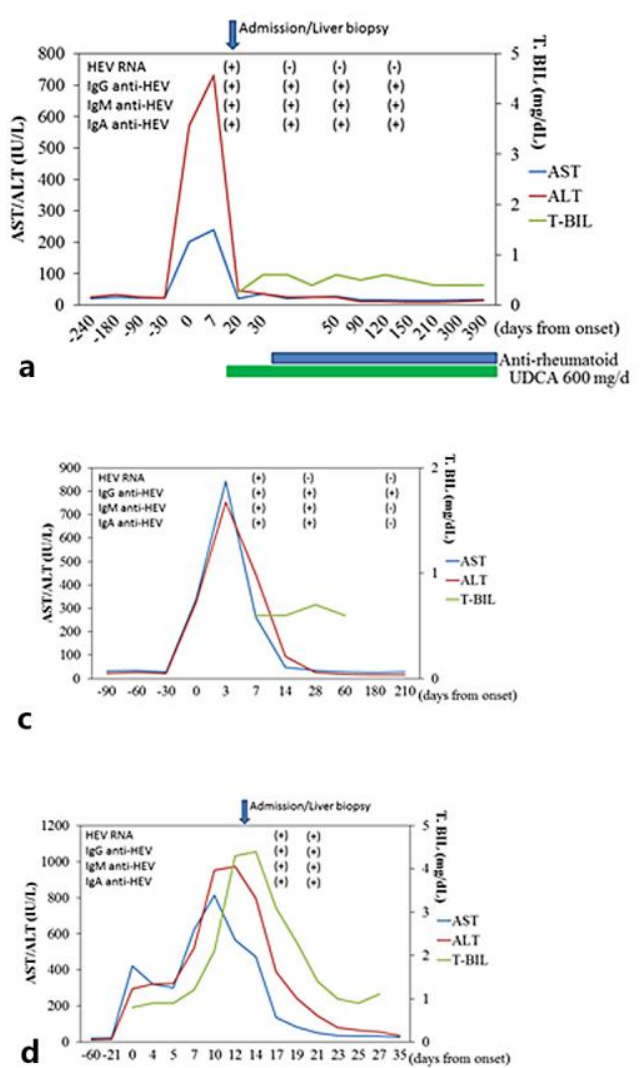

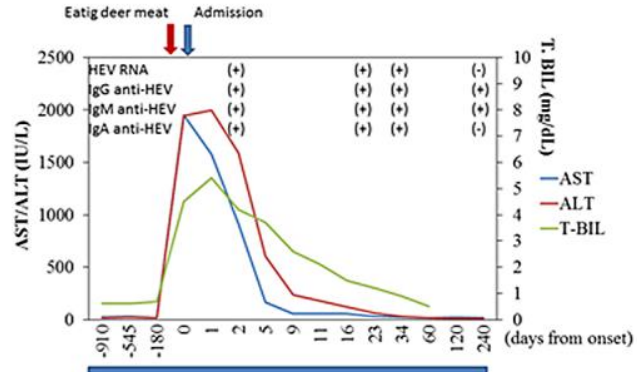

b Acetylsalicylic acid

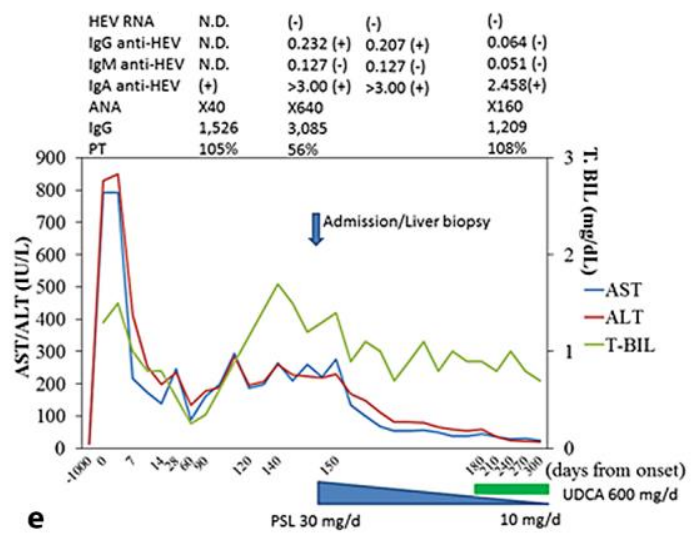

Fig. 1. Clinical course of 5 patients with HEV infection in the present study. a Case 1. b Case 2. c Case 3. d Case 4. e Case 5. Cases 1, 3, and 4 visited a hospital for their RA. Cases 1-4 were positive for HEV RNA at least at one time point. Samples from cases 2-4 contain HEV genotype $3 \mathrm{~b}$ determined based on an analysis of the 412-nt ORF2 sequence [20]. As the HEV RNA level was low titer in case 1, the sample from case 1 contains HEV genotype 3 determined based on an analysis of the 97-nt ORF2/3 sequence [20]. In case 5, we did not detect HEV RNA, but we diagnosed this case as HEV infection according to changes in titers of anti-HEV antibodies. AST = Aspartate transaminase; ALT = alanine transaminase; T-BIL = total bilirubin; UDCA = ursodeoxycholic acid; ANA = anti-nuclear antibody; $\mathrm{PT}=$ prothrombin time; $\mathrm{PSL}=$ prednisolone. 


\begin{tabular}{|c|c|c|}
\hline \multirow{2}{*}{$\begin{array}{l}\text { Case Reports in } \\
\text { Gastroenterology }\end{array}$} & \multicolumn{2}{|c|}{ Case Rep Gastroenterol 2015;9:317-326 } \\
\hline & DOI: $10.1159 / 000441387$ & $\begin{array}{l}\text { @ } 2015 \text { The Author(s). Published by S. Karger AG, Basel } \\
\text { www.karger.com/crg }\end{array}$ \\
\hline
\end{tabular}

Kanda et al:: Recent Trend of Hepatitis E Virus Infection in Chiba Area, Japan: 3 of 5 Cases with Rheumatoid Arthritis

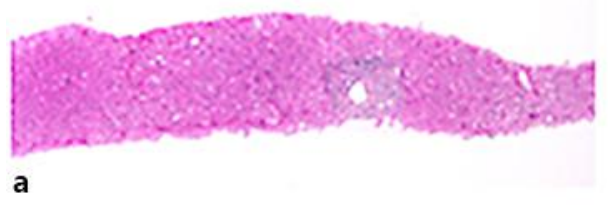

C
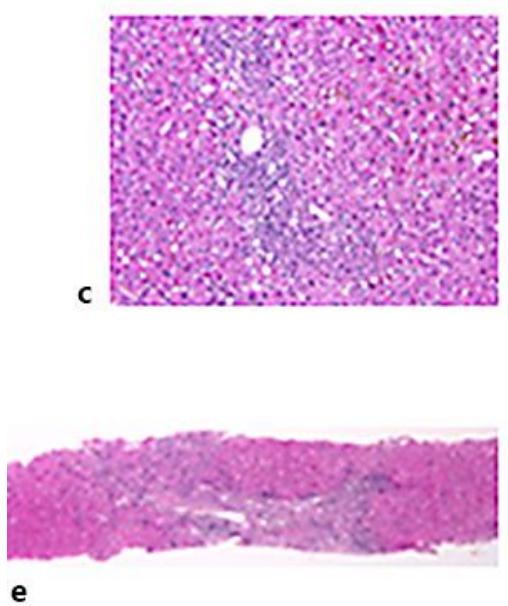

e

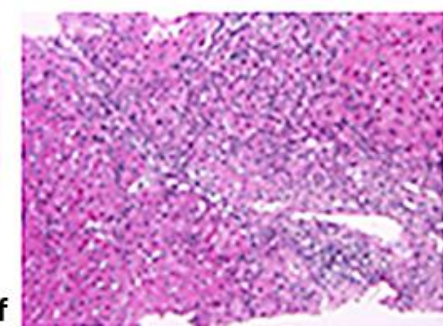

d
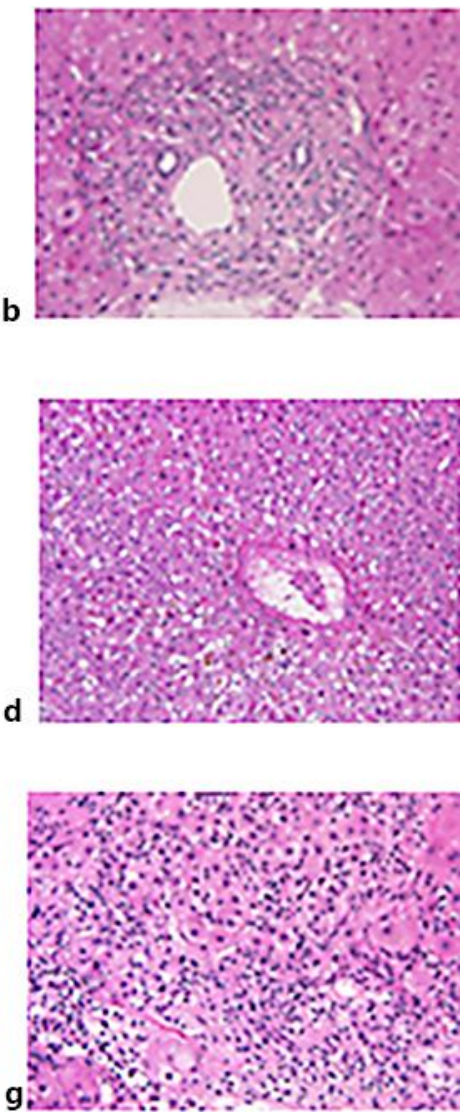

Fig. 2. Liver biopsy findings in cases 1,4 , and 5. In case 1, the hepatic architecture was preserved (a HE, $\times 40$ ), and findings were compatible to Scheuer stage I of PBC (b HE, $\times 100$ ). In case 4 , the hepatic architecture was preserved, and marked inflammation in periportal areas (c HE, $\times 100)$ and centrilobular necrosis (d HE, $\times 100$ ) were observed, indicating acute hepatitis. In case 5 , a liver biopsy showed a partly preserved hepatic architecture but no cirrhosis (e HE, $\times 40$ ). Marked inflammation including rosette formation in the periportal area $(\mathbf{f H E}, \times 100)$ and plasma cell infiltration $(\mathrm{g} \mathrm{HE}, \times 100)$ were observed, suggesting autoimmune hepatitis. 